\title{
Assessing the Medical Emergency Preparedness of Dental Faculty, Residents, and Practicing Periodontists: An Exploratory Study
}

\author{
Tatiana de Bedout, Kyle Kramer, Steven Blanchard, Yusuke Hamada, George J. Eckert, \\ Gerardo Maupome, Vanchit John
}

Abstract: With the increased number of elderly and medically compromised individuals receiving dental care and the presence of systemic comorbidities and associated treatment modalities in this patient population, it is imperative that dentists be prepared to manage a variety of medical emergencies. The aim of this study was to assess the knowledge of and preparedness to manage common medical emergencies of cohorts of practicing periodontists, specialty residents, and faculty members, both for comparative purposes and as an aid to refining a dental school's standardized case scenarios. The study, conducted in 2017, was designed for four groups of randomly selected participants with at least 20 in each group; the actual number of voluntary participants was 28 private practice periodontists, 22 residents in specialty programs, 21 specialist faculty members, and 24 general practice faculty members. Participants were asked to evaluate ten clinical emergency cases and identify the diagnosis and indicated intervention for each. Groups were also evaluated for differences among correct responses for each case. Overall, there were no statistically significant differences for number of correct diagnoses or interventions among the four groups. However, several cases had varying degrees of incorrect diagnoses and management across all groups. Participants who had recently graduated or were still in school were able to treat cases appropriately more often than the other participants. Further refinement of cases to assess provider preparedness to correctly diagnose and manage medical emergencies is needed, specifically establishing case-specific features and addressing areas of potential confusion before the cases are used for educational purposes.

Tatiana de Bedout, DDS, is in private practice in Indianapolis, IN; this study was conducted while she was a resident, Department of Periodontics and Allied Dental Program, Indiana University School of Dentistry; Kyle Kramer, DDS, MS, is Assistant Clinic Professor of Dental Anesthesiology, Department of Oral Surgery and Hospital Dentistry, Indiana University School of Dentistry; Steven Blanchard, DDS, MS, is Director of Graduate Periodontics, Indiana University School of Dentistry; Yusuke Hamada, DDS, MSD, is Clinical Assistant Professor, Department of Periodontics and Allied Dental Program, Indiana University School of Dentistry; George J. Eckert, MAS, is Biostatistician Supervisor, Department of Biostatistics, Indiana University School of Medicine; Gerardo Maupome, BDS, MSc, PhD, is Professor, Department of Social and Behavioral Sciences, and Associate Dean of Research, Richard M. Fairbanks School of Public Health, Indiana University; and Vanchit John, DDS, MSD, is Chair, Department of Periodontics and Allied Dental Programs, Indiana University School of Dentistry. Direct correspondence to Dr. Vanchit John, Department of Periodontics and Allied Dental Programs, Indiana University School of Dentistry, 1121 West Michigan Street, Indianapolis, IN 46202; 317-274-5124; vjohn@iu.edu.

Keywords: dental education, medical emergencies, patient safety, medical errors, educational methodology, case-based learning

Submitted for publication 7/29/17; accepted 11/11/17

doi: $10.21815 / J D E .018 .058$

$\mathrm{G}$ eneral improvement in the quality of health care has increased the life expectancy of the U.S. population seeking dental care. Accordingly, dentists are treating a growing number of elderly and medically compromised patients. ${ }^{1}$ It is therefore important that oral health care providers be prepared to manage a variety of medical emergencies. While serious or major medical emergencies rarely occur in dental practices, being prepared to satisfactorily manage a medical emergency is critical, as it may, in fact, be life-saving. Utilizing self-reported data gathered from a survey of dentists, Muller et al. found that $57 \%$ of the responding dentists reported up to three emergencies in a 12-month period, with $36 \%$ reporting up to ten emergencies. ${ }^{2}$ In their study, vasovagal syncope was the most frequent emergency; 42 severe life-threatening events were reported among the treated patients; and $92 \%$ of the respondents had taken part in emergency training following graduation (23\% participated once and $68 \%$ more than once). Vasovagal syncope has also been reported as the most frequent event in other studies, with nonsyncopal events being less frequent. ${ }^{3} \mathrm{Al}-$ Sebaei et al. reported the distribution of emergencies in private 
dental offices as vasovagal syncope (63\%), angina $(12 \%)$, hypoglycemia (8\%), epileptic seizures $(8 \%)$, choking (5\%), asthma (3\%), and anaphylaxis (1\%). ${ }^{4}$

Medical emergencies are most likely to occur during periods of stress, such as the times surrounding the administration of local anesthesia, tooth extraction, or stimulating endodontic procedures. ${ }^{5-7}$ Medical emergencies often arise suddenly and unexpectedly, so it is imperative that dentists be able to rapidly recognize the onset of an emergency, diagnose the condition, and provide the indicated management.

There are limited data on the level of preparedness among oral health providers in the U.S. as well as globally. Al-Sebaei et al.'s study of private dental offices in Saudi Arabia found a deficiency in personnel training and in the availability of drugs and emergency equipment. ${ }^{4} \mathrm{~A}$ study conducted in India looked at the availability of medical emergency drugs in dental offices and dentists' level of knowledge to manage medical emergencies. ${ }^{8}$ The authors concluded that almost all of the participating dentists (98\%) did inquire about their patients' medical history, but only $12 \%$ documented that information. In addition, only $38.4 \%$ recorded vital signs prior to dental procedures, and only $7.6 \%$ reported having attended any continuing education courses on emergency training or management programs. Kieser and Herbison found a high level of anxiety in dental students in New Zealand when dealing with medical emergencies. ${ }^{9}$ In their study, Fast et al. reported that if an emergency occurred in the dental office, $66 \%$ of practicing dentists believed they knew what their legal responsibilities were, $85 \%$ believed they knew what their professional responsibilities were, and $64 \%$ were confident that the dental team would provide appropriate care. ${ }^{10}$ However, only $44 \%$ were comfortable with their present state of emergency preparedness, and 4\% indicated they would rather not know too much or not have emergency equipment in the office as they would otherwise likely be held liable. Anders et al. reported that the incidence of medical emergencies at a dental school was 164 events per million patient visits; most emergencies were related to cardiovascular events, syncope, complications with local anesthesia, and hypoglycemia. ${ }^{11}$

To help clinicians with the preparedness to handle medical emergencies, regular mock emergency drills should be considered. Those drills would help the dentist and staff members become more confident in their roles during emergency situations, improving their level of knowledge and overall preparedness.
At Indiana University School of Dentistry (IUSD) in the Department of Periodontics and Allied Dental Programs, there has been an emphasis on medical emergency preparedness since 2010. Monthly emergency drills have been conducted along with an annual written assessment to help evaluate the level of preparedness among the residents, staff, and department faculty. While it is intended that these drills will continue, we sought to use the program as a research opportunity to refine key aspects of the case scenarios used in the ongoing monthly emergency drills. Such a study would also be useful because comparative assessment of preparedness among generalists, specialists, and residents training to become specialists had not been previously reported. The aim of this study was thus to assess the knowledge of and preparedness to manage common medical emergencies of cohorts of practicing periodontists, specialty residents, and faculty members, both for comparative purposes and as an aid to refining the dental school's standardized case scenarios.

\section{Methods}

The IUSD Institutional Review Board approved this study (\#1702238703). Prior to the study being conducted and IRB submission, a peer review of the questionnaire and cases was conducted to assess their content validity. The participants in this review were four general dentists and two prosthodontists with extensive clinical experience. Those individuals were not included in the study. Based on their responses, the case scenarios were refined, and a multiple-choice format was chosen for the survey design.

The study, conducted in 2017, was designed for four groups of study participants with at least 20 individuals in each group: general practice faculty members (full- and part-time), specialist faculty members (full-time and adjunct periodontists, prosthodontists, endodontists, and orthodontists), residents in the Departments of Periodontics, Prosthodontics, Endodontics, and Orthodontics, and full-time periodontists in practice (alumni of IUSD graduate periodontics program). With a sample size of 20 per group, the study would have an $80 \%$ power to detect a difference of $45 \%$ or less for total correct scores between any two groups for the individual clinical cases.

A list of potential study participants was randomly selected. Potential participants were contacted to ascertain their availability and willingness to participate in the study. Requests for participation were 
sent to ten people at a time from each group. They were contacted via email and informed that their participation was voluntary and they could withdraw at any point if needed. The selection process continued until there were at least 20 participants for each group.

The study participants were asked to complete a survey identifying their level of training and then to evaluate ten clinical medical emergency cases. Fifteen questions asked about the participants' background in dentistry, their involvement with medical emergencies in dental settings, and whether they had taken courses on medical emergencies preparedness. On the cases, participants were asked to provide a diagnosis via an open-text response for each scenario, which was subsequently categorized as either acceptable or not. They were also asked to select an appropriate intervention from a list of four multiple-choice options created specifically for each scenario. The responses were entered using Google Drive, which allowed each subject to answer all the questions without having to add any confidential information. The approach adhered closely to strategies used as a professional standard for high-fidelity, real-life clinical cases that challenge clinicians to self-assess their knowledge, training, and responses. The correct diagnosis and best plan of intervention for each scenario were reviewed and agreed upon by the research committee members for the project.

The total score for each participant was calculated as the percentage of correct responses across all ten cases, separately classifying diagnoses and interventions. Comparisons between the groups for differences between the total scores were made using nonparametric Kruskal-Wallis tests. In addition to the total scores, the groups were compared for differences in the percentage of correct responses for each individual case using Fisher's exact tests. A 5\% significance level was used for all tests.

\section{Results}

A total of 95 dentists participated in the study: 28 private practice periodontists, 24 general practitioner faculty members, 22 residents in specialty programs, and 21 specialist faculty members. The total amount of time to respond to the background and case questions was no longer than 60 minutes, typically taking participants between 15 and 20 minutes.

Most participants $(79 \%)$ had received their dental training in the U.S., and their graduation year or anticipated graduation year for the residents ranged from 1967 through 2019. The majority (75\%) indicated they had either witnessed or managed syncope while practicing dentistry. Other medical emergencies they had witnessed or managed while practicing dentistry either in their private offices or at the dental school are shown in Figure 1. The majority (69\%) said they felt comfortable managing medical emergencies in a dental setting. Only about half $(53 \%)$ had been trained to administer minimalmoderate sedation. Most of the participants indicated that they recorded blood pressure before starting any treatment $(85 \%)$, with $76 \%$ recording pulse rate. However, $16 \%$ did not record any vital signs prior to starting any dental procedures.

The most common emergency management interventions used at the private office or the dental school were supplemental oxygen $(73 \%)$, oral glucose administration (58\%), and activation of Emergency Medical Services (EMS) (46\%); other interventions are shown in Figure 2. When asked if they practiced medical emergency response preparedness regularly, most (83\%) indicated that they did not or very seldom did so. It was only the periodontics residents and some of the periodontists who practiced monthly medical emergency drills.

On the ten medical emergency cases, each group had approximately eight correct diagnoses and six or seven correct interventions (Table 1). There were no significant differences among the four groups for number of correct diagnoses $(\mathrm{p}=0.68)$ or number of correct interventions $(\mathrm{p}=0.31)$.

When we analyzed the clinical cases individually, most of the case scenarios did not show statistically significant differences among the participant groups, but three did. There were no significant differences among the four groups for diagnosis or management (Table 2) for Case 1 (asthma) $(\mathrm{p}=0.46$ for diagnosis, $\mathrm{p}=0.39$ for management), Case 2 (hyperventilation) $(\mathrm{p}=0.28$ for diagnosis, $\mathrm{p}=0.50$ for management), Case 3 (seizure) ( $\mathrm{p}=1.00$ for diagnosis, $\mathrm{p}=0.24$ for management), Case 4 (mild to moderate allergic reaction) $(\mathrm{p}=0.76$ for diagnosis, $\mathrm{p}=0.89$ for management), Case 5 (choking) ( $\mathrm{p}=0.90$ for diagnosis, $\mathrm{p}=0.92$ for management), Case 6 (hypoglycemia) $(p=0.38$ for diagnosis, $p=0.77$ for management), or Case 10 (myocardial infarction) $(\mathrm{p}=0.88$ for diagnosis, $\mathrm{p}=0.67$ for management).

However, on Case 7 (cardiac arrest), the residents had a significantly lower percentage of correct diagnoses $(64 \%, \mathrm{p}=0.0419)$ than the private practice periodontists $(89 \%, \mathrm{p}=0.0086)$ and the general practitioner faculty $(96 \%)$, with no other differences among the groups ( $>0.16$ ) (Table 2). Also, the private practice periodontists had a significantly 


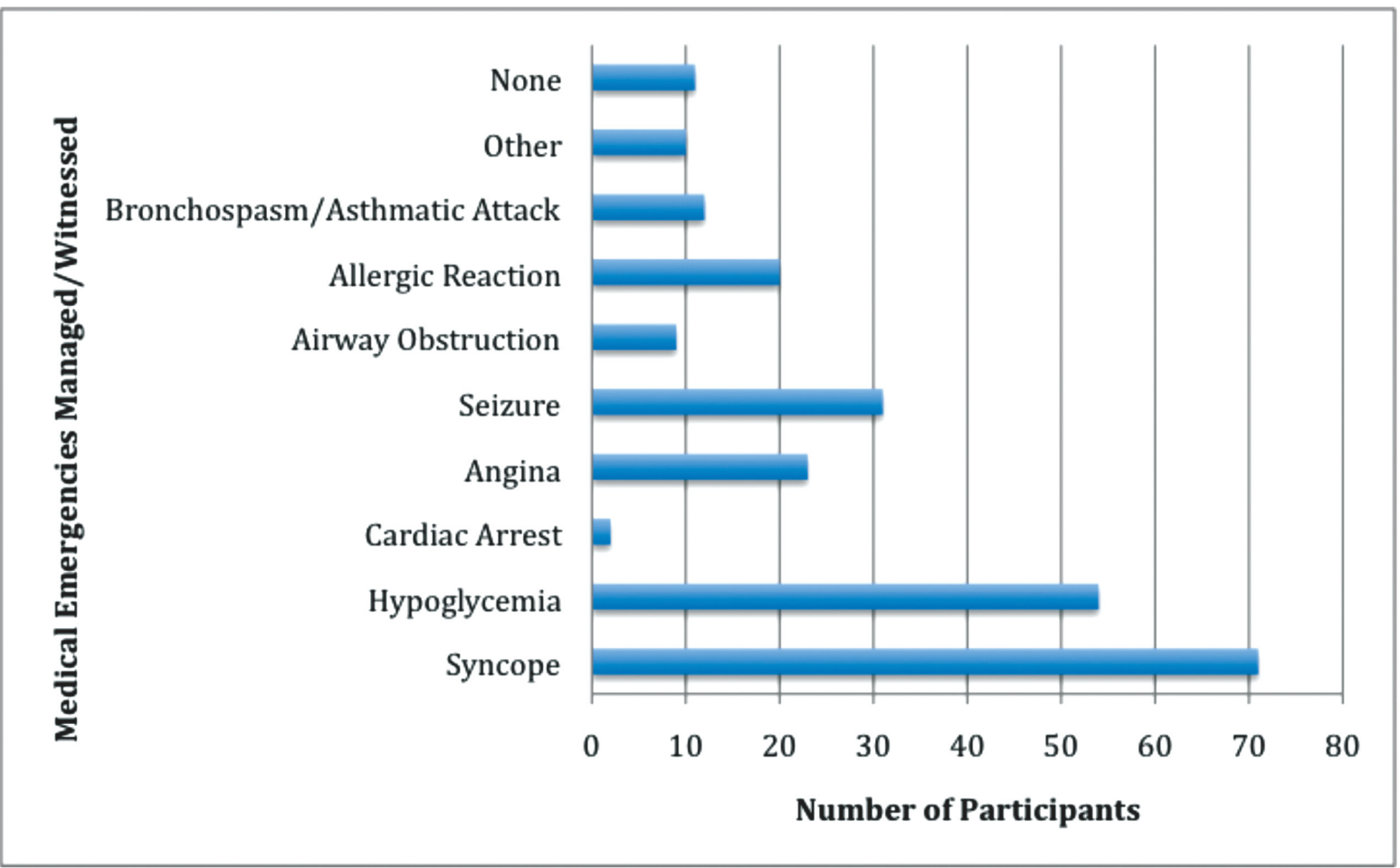

Figure 1. Number of participants who had managed or witnessed medical emergencies $(\mathrm{N}=95)$

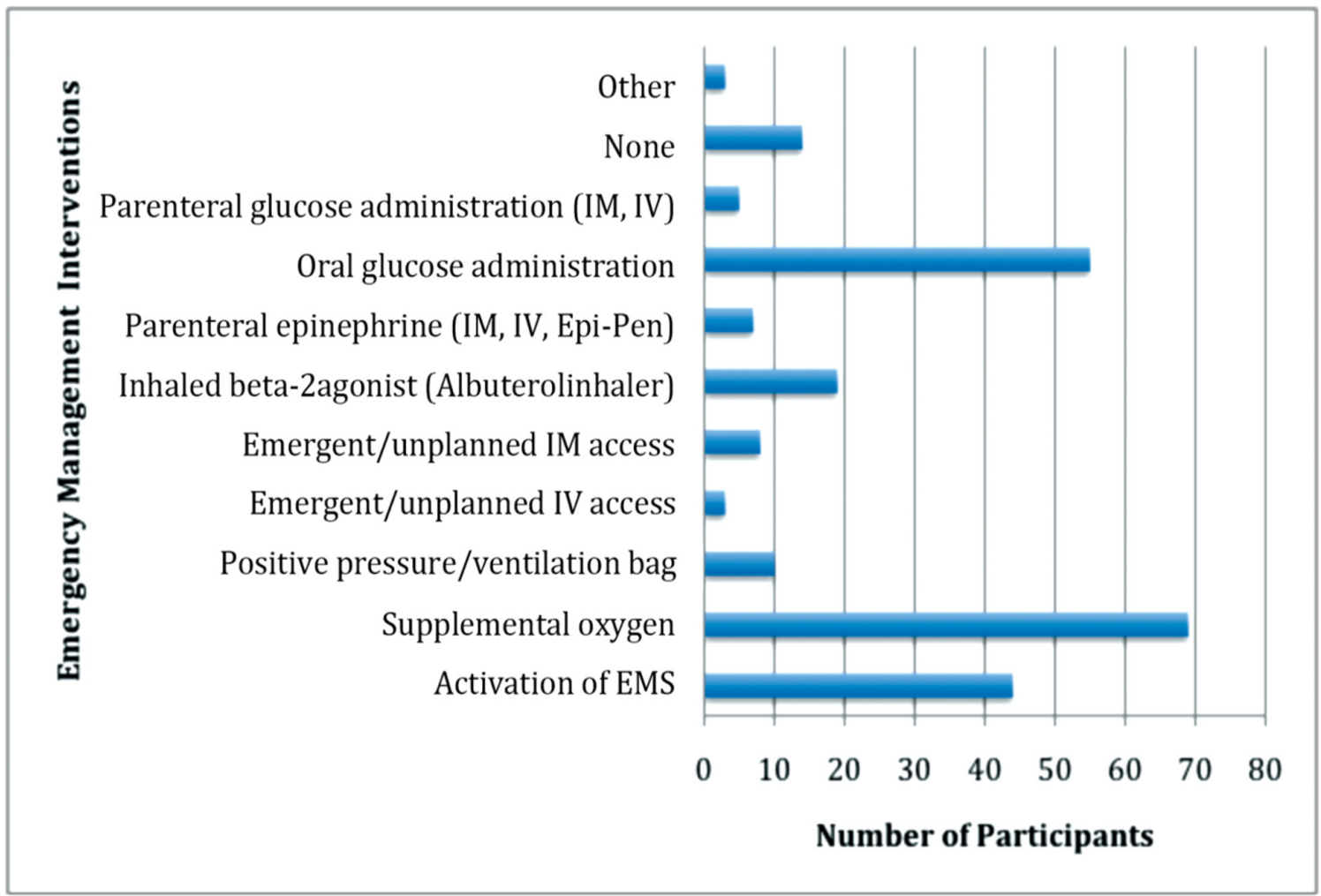

Figure 2. Number of participants who had used common emergency management interventions $(\mathrm{N}=95)$ 
higher correct plan of intervention $(82 \%)$ than the specialist faculty $(38 \%)(\mathrm{p}=0.0025)$, with no other differences among the groups $(\mathrm{p}>0.07)$. The most accurate treatment was to begin CPR with 30 chest compressions to a depth of two inches, which $66 \%$ of the participants answered correctly (Figure 3 ). On Case 8 (anaphylaxis), the general practitioner faculty members' performance for diagnosis was statistically significant (100\%) compared to the residents $(73 \%)$ $(\mathrm{p}=0.008)$, with no other differences among groups $(p>0.09)$. There were no significant differences among the four groups with respect to the intervention for Case $8(\mathrm{p}=0.65)$. On Case 9 (syncope), the private practice periodontists had significantly better diagnoses $(96 \%)$ than the specialist faculty $(67 \%)$, with no other differences among groups ( $p>0.07$ ). There were no significant differences among the four groups for syncope management $(\mathrm{p}=0.25)$.

The overall average of correct responses based on the years when the participants completed or anticipated completing their specialty training program or as a general dentist is shown in Figure 4.

Table 1. Correct diagnoses and interventions out of ten cases by group: mean (SD), minimum, maximum numbers

\begin{tabular}{|c|c|c|c|c|c|}
\hline Total Correct & Group & $\mathrm{N}$ & Mean (SD) & Min & Max \\
\hline \multirow[t]{4}{*}{ Diagnoses } & Private practice periodontists & 28 & $8.3(1.0)$ & 6 & 10 \\
\hline & General practitioner faculty & 24 & $8.3(0.8)$ & 7 & 10 \\
\hline & Residents & 22 & $7.7(1.6)$ & 4 & 10 \\
\hline & Specialist faculty & 21 & $8.0(1.4)$ & 4 & 10 \\
\hline \multirow[t]{4}{*}{ Interventions } & Private practice periodontists & 28 & $7.1(1.6)$ & 5 & 10 \\
\hline & General practitioner faculty & 24 & $6.5(1.9)$ & 3 & 10 \\
\hline & Residents & 22 & $7.0(1.6)$ & 3 & 10 \\
\hline & Specialist faculty & 21 & $6.3(1.7)$ & 3 & 9 \\
\hline
\end{tabular}

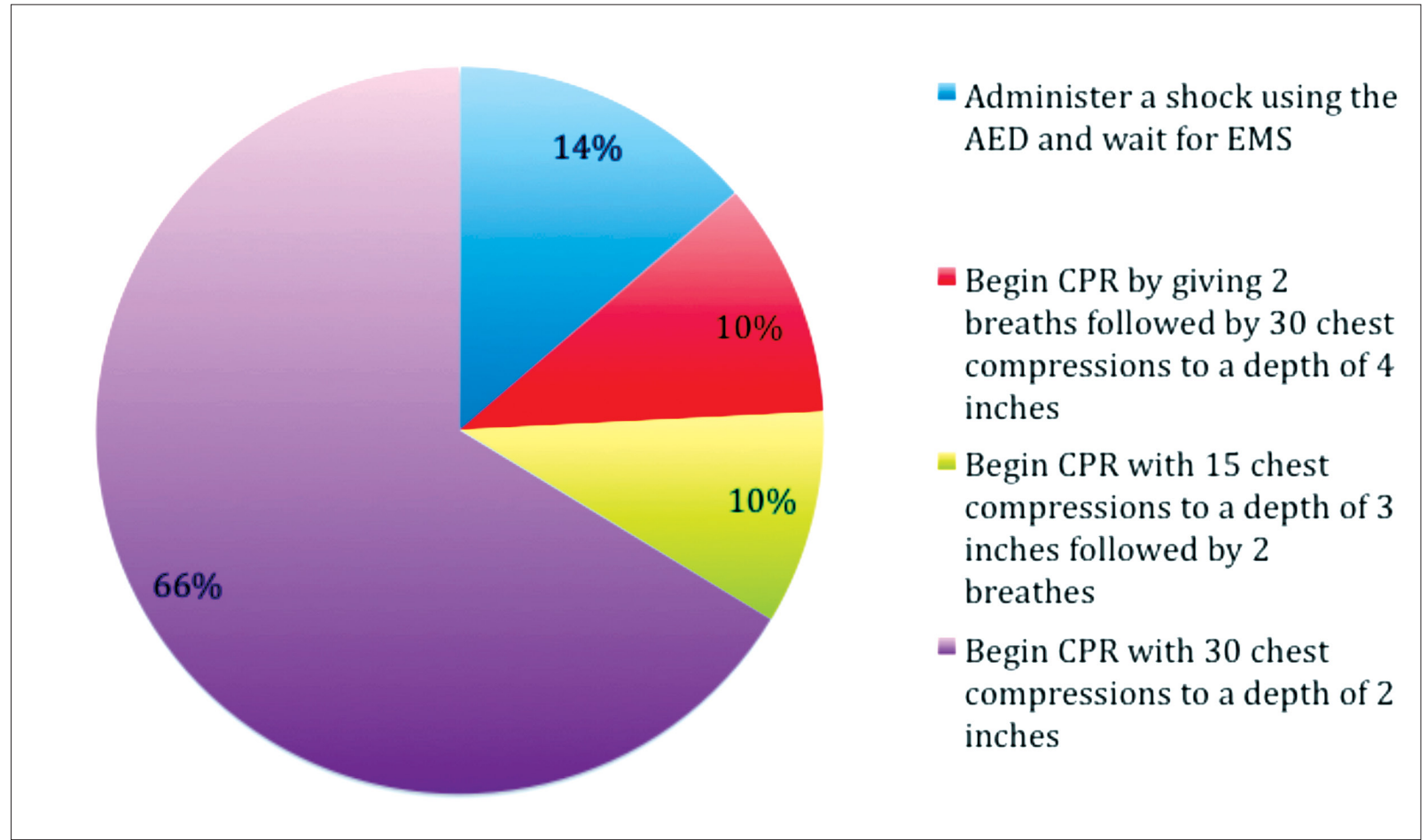

Figure 3. Responses on best basic intervention step for management of cardiac arrest (Case 7), by percentage of total participants $(\mathrm{N}=95)$

Note: Correct response was "Begin CPR with 30 chest compressions to a depth of 2 inches." 
Table 2. Correct and incorrect diagnoses and interventions on each case by number and percentage of each group

\begin{tabular}{|c|c|c|c|c|c|}
\hline \multirow{2}{*}{ Case } & \multirow[b]{2}{*}{ Group } & \multicolumn{2}{|c|}{ Diagnosis } & \multicolumn{2}{|c|}{ Intervention } \\
\hline & & Correct & Incorrect & Correct & Incorrect \\
\hline \multirow[t]{4}{*}{ 1: Asthma } & PPP & $20(71 \%)$ & $8(29 \%)$ & $16(57 \%)$ & $12(43 \%)$ \\
\hline & GP faculty & $19(79 \%)$ & $5(21 \%)$ & $13(54 \%)$ & $11(46 \%)$ \\
\hline & Residents & $17(77 \%)$ & $5(23 \%)$ & $12(55 \%)$ & $10(45 \%)$ \\
\hline & SP faculty & $19(90 \%)$ & $2(10 \%)$ & $16(76 \%)$ & $5(24 \%)$ \\
\hline \multirow[t]{4}{*}{ 2: Hyperventilation } & PPP & $19(68 \%)$ & $9(32 \%)$ & $22(79 \%)$ & $6(21 \%)$ \\
\hline & GP faculty & $14(58 \%)$ & $10(42 \%)$ & $18(75 \%)$ & $6(25 \%)$ \\
\hline & Residents & $9(41 \%)$ & $13(59 \%)$ & $19(86 \%)$ & $3(14 \%)$ \\
\hline & SP faculty & $13(62 \%)$ & $8(38 \%)$ & $14(67 \%)$ & $7(33 \%)$ \\
\hline \multirow[t]{4}{*}{ 3: Seizure } & PPP & $28(100 \%)$ & 0 & $19(68 \%)$ & $9(32 \%)$ \\
\hline & GP faculty & $24(100 \%)$ & 0 & $11(46 \%)$ & $13(54 \%)$ \\
\hline & Residents & $22(100 \%)$ & 0 & $16(73 \%)$ & $6(27 \%)$ \\
\hline & SP faculty & $21(100 \%)$ & 0 & $12(57 \%)$ & $9(43 \%)$ \\
\hline \multirow[t]{4}{*}{ 4: Mild to moderate allergic reaction } & PPP & $19(68 \%)$ & $9(32 \%)$ & $27(96 \%)$ & $1(4 \%)$ \\
\hline & GP faculty & $18(75 \%)$ & $6(25 \%)$ & $23(96 \%)$ & $1(4 \%)$ \\
\hline & Residents & $18(82 \%)$ & $4(18 \%)$ & $22(100 \%)$ & 0 \\
\hline & SP faculty & $16(76 \%)$ & $5(24 \%)$ & $20(95 \%)$ & $1(5 \%)$ \\
\hline \multirow[t]{4}{*}{ 5: Choking } & PPP & $26(93 \%)$ & $2(7 \%)$ & $15(54 \%)$ & $13(46 \%)$ \\
\hline & GP faculty & $23(96 \%)$ & $1(4 \%)$ & $15(63 \%)$ & $9(38 \%)$ \\
\hline & Residents & $21(95 \%)$ & $1(5 \%)$ & $12(55 \%)$ & $10(45 \%)$ \\
\hline & SP faculty & $21(100 \%)$ & 0 & $12(57 \%)$ & $9(43 \%)$ \\
\hline \multirow[t]{4}{*}{ 6: Diabetic with hypoglycemic episode } & PPP & $21(75 \%)$ & $7(25 \%)$ & $23(82 \%)$ & $5(18 \%)$ \\
\hline & GP faculty & $15(63 \%)$ & $9(38 \%)$ & $20(83 \%)$ & $4(17 \%)$ \\
\hline & Residents & $18(82 \%)$ & $4(18 \%)$ & $18(82 \%)$ & $4(18 \%)$ \\
\hline & SP faculty & $13(62 \%)$ & $8(38 \%)$ & $15(71 \%)$ & $6(29 \%)$ \\
\hline \multirow[t]{4}{*}{ 7: Cardiac arrest } & PPP & $25(89 \%)$ & $3(11 \%)$ & $23(82 \%)$ & $5(18 \%)$ \\
\hline & GP faculty & $23(96 \%)$ & $1(4 \%)$ & $16(67 \%)$ & $8(33 \%)$ \\
\hline & Residents & $14(64 \%)$ & $8(36 \%)$ & $16(73 \%)$ & $6(27 \%)$ \\
\hline & SP faculty & $17(81 \%)$ & $4(19 \%)$ & $8(38 \%)$ & $13(62 \%)$ \\
\hline \multirow[t]{4}{*}{ 8: Anaphylaxis } & PPP & $26(93 \%)$ & $2(7 \%)$ & $22(79 \%)$ & $6(21 \%)$ \\
\hline & GP faculty & $24(100 \%)$ & 0 & $17(71 \%)$ & $7(29 \%)$ \\
\hline & Residents & $16(73 \%)$ & $6(27 \%)$ & $19(86 \%)$ & $3(14 \%)$ \\
\hline & SP faculty & $18(86 \%)$ & $3(14 \%)$ & 17 (81\%) & $4(19 \%)$ \\
\hline \multirow[t]{4}{*}{ 9: Syncope } & PPP & $27(96 \%)$ & $1(4 \%)$ & $25(89 \%)$ & $3(11 \%)$ \\
\hline & GP faculty & $19(79 \%)$ & $5(21 \%)$ & $18(75 \%)$ & $6(25 \%)$ \\
\hline & Residents & $17(77 \%)$ & $5(23 \%)$ & $18(82 \%)$ & $4(18 \%)$ \\
\hline & SP faculty & $14(67 \%)$ & $7(33 \%)$ & $14(67 \%)$ & $7(33 \%)$ \\
\hline \multirow[t]{4}{*}{ 10: Myocardial infarction } & PPP & $22(79 \%)$ & $6(21 \%)$ & $8(29 \%)$ & $20(71 \%)$ \\
\hline & GP faculty & $21(88 \%)$ & $3(13 \%)$ & $5(21 \%)$ & $19(79 \%)$ \\
\hline & Residents & $18(82 \%)$ & $4(18 \%)$ & $3(14 \%)$ & $19(86 \%)$ \\
\hline & SP faculty & $17(81 \%)$ & $4(19 \%)$ & $5(24 \%)$ & $16(76 \%)$ \\
\hline
\end{tabular}

PPP=private practice periodontists $(\mathrm{N}=28)$; GP faculty=general practitioner faculty members $(\mathrm{N}=24)$; Residents in specialty programs $(\mathrm{N}=22) ; \mathrm{SP}$ faculty=specialist faculty members $(\mathrm{N}=21)$

The groups consisted of those with graduation years 1967-80 (13), 1981-90 (13), 1991-2000 (20), 2004-10 (12), and 2012-19 (31). Six participants answered this question incorrectly, so the total number on this item was 89 .
In reviewing differences among the study groups by years of experience, we found that statistical analysis of the data was challenging as graduation year was confounded with participant groups. The residents were all in the 2012-19 graduation year 


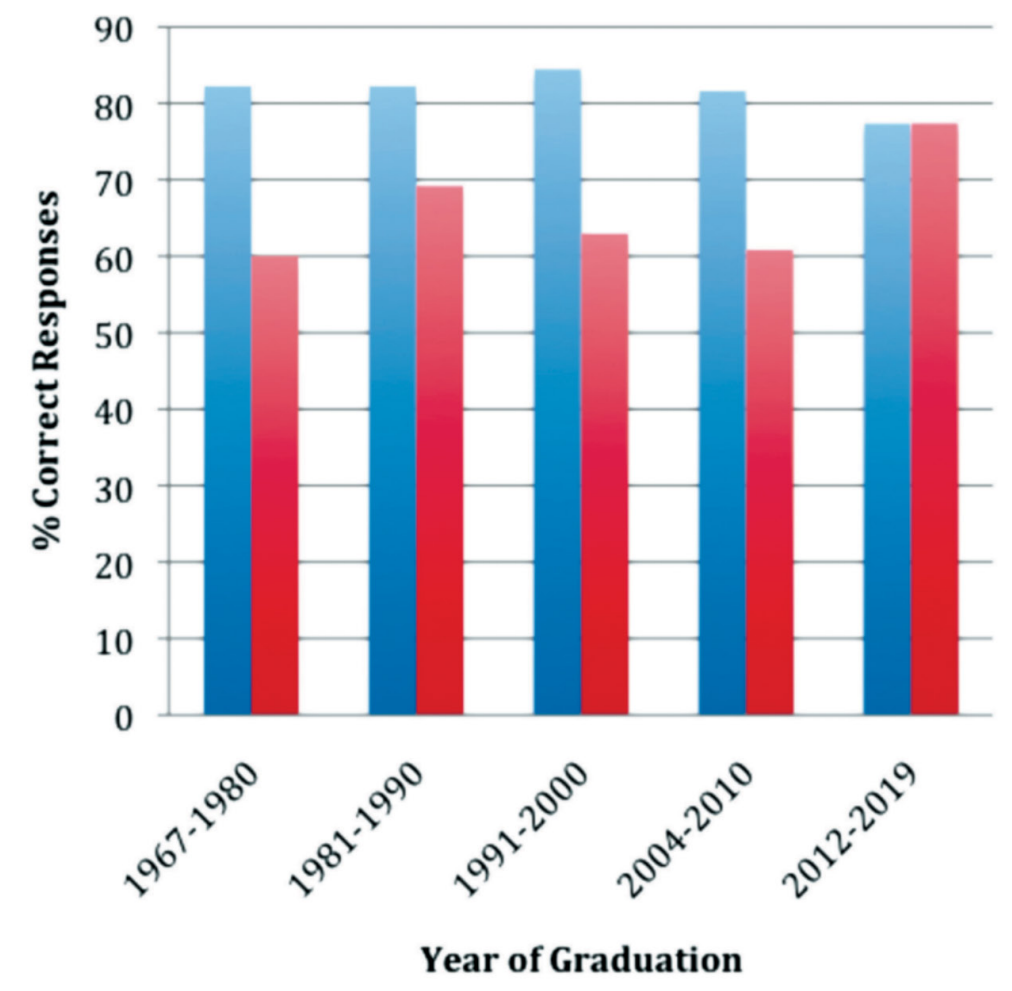

Ave \% Correct Diagnosis

Ave \% Correct Treatment

Figure 4. Average correct responses on total cases, by participants' year of graduation

Note: Total numbers of participants by year of graduation range were: 1967-80 (13), 1981-90 (13), 1991-2000 (20), $2004-10$ (12), and 2012-19 (31).

group. The general practice faculty members were primarily in the 1967-80 and 1981-90 groups. The alumni and specialist faculty members were a mix of years but focused more towards the middle years. This clustering made it very difficult to distinguish differences as being due to group or graduation year. For participants graduating in 1967-80, 50\% felt comfortable dealing with a medical emergency, and only $30 \%$ were trained to administer minimalmoderate sedation. For participants graduating in $1981-90,85 \%$ felt comfortable treating the medical emergencies, and only $7 \%$ were trained to administer minimal-moderate sedation. For participants graduating in 1991-2000, 55\% felt comfortable with medical emergencies, and $60 \%$ were trained to administer minimal-moderate sedation. For participants graduating in 2004-10, 67\% felt comfortable with medical emergencies, and $58 \%$ were trained for minimalmoderate sedation. The group graduating in 2012-19 had the most training with minimal-moderate sedation at $77 \%$, and $75 \%$ of this group felt comfortable with medical emergencies.
While most participants were able to correctly diagnose the case scenarios, those who could best identify the correct interventions were those in the most recent graduation year group (2012-19). Overall, there were no statistically significant differences among graduation periods in dealing with medical emergencies $(p=0.35)$, training for administering minimal-moderate sedation $(p>0.06)$, number of correct diagnoses $(p=0.75)$, or number of correct interventions $(\mathrm{p}=0.34)$.

\section{Discussion}

To our knowledge, no previous studies have compared the knowledge of dental specialty residents, general practice faculty, specialist faculty, and specialist practitioners (periodontists) in medical emergency preparedness in a dental setting in the U.S. The participants were selected based on their being practicing clinicians, both general dentists and specialists. Residents were chosen from the same departments as the specialist faculty. 
Being able to identify as well as manage a medical emergency is crucial for the safety of patients. In our study, most of the dental professionals surveyed had managed a vasovagal syncopal event during their professional careers. This finding was consistent with prior studies in identifying syncope as the most common medical emergency; however, our study found hypoglycemia to be a more common finding than in those previous studies..$^{4-7,12}$ The reasons for this are merely speculative but since there were more periodontists (clinicians and residents) in the group overall, the patient pool could include those presenting with a history of diabetes mellitus as there is an established relationship between periodontal disease and diabetes mellitus. ${ }^{13}$ In addition, since some of the patients were having periodontal surgery, they may have not eaten a meal prior to the procedure while inadequately reporting their information to the clinician.

Taken as a group, most of the participants in our study performed reasonably well by correctly diagnosing and managing the clinical case studies overall. The mean for correct diagnosis of individual groups ranged from 7.7 to 8.3 on the ten cases. Likewise, the mean for correct interventions ranged from 6.3 to 7.1 , with no statistically significant differences. However, a few cases were inadequately diagnosed and managed across all groups.

While all groups generally performed well in diagnosing the cases, some showed a lower proficiency in identifying an appropriate plan of action. On the cardiac arrest case, most groups were able to correctly diagnose the emergency, but less so for choosing the appropriate management. Correct management is essential because, according to the American Heart Association (AHA), a person may become brain dead or die just four to six minutes after cardiac arrest. ${ }^{14}$ With this in mind and the fact that not everyone is trained to use an automated external defibrillator or has taken continuing education (CE) courses on medical emergencies, the correct, rapid intervention could be the difference between life and death for a patient. We found that those participants who had recently graduated (as early as 2012) or were still in school were able to treat cases appropriately more often than the other participants. This finding indicates that training currently provided by the school has added a valuable component to the treatment of medical emergencies.

The study also found a large difference in the number of CE courses or classes dedicated to medical emergency training each participant had taken.
Currently, there is no specified number of mock drills or CE courses defined as optimal for preparing clinicians for medical emergencies. While the American Dental Association (ADA) cannot mandate that private dental offices have specific types of emergency protocols, drugs, or equipment available, it does recommend that one "should" provide "appropriate training" to staff and encourages a regular review of mock drills at least annually. ${ }^{15}$ However, it is imperative that dental practitioners make sure that their offices are equipped with appropriate emergency kits and that the clinicians update their knowledge base with regular $\mathrm{CE}$ that includes management of common medical emergencies.

There were several limitations to this study. The study included a small sample size for each group, although the number in each group did exceed that defined in the power analysis. Another possible limitation is that the clinical cases were graded based on a multiple-choice response. It is unclear whether the participants' comprehension would be more accurately assessed with open-ended or short answer questions that required them to provide the answer. Although our testing platform and the battery of case scenarios were standardized for evaluation in identical circumstances for all participants, there is a possibility that some participants may have differentially interpreted the cases, questions, or answer options leading to incorrect responses. In addition, this exploratory study included only clinicians who had an affiliation with IUSD. Future studies should aim to include a larger sample size and to explore further analysis, refinement, and clarification of the testing items. One option is to begin by assessing the "why" not only the "how" information processing should start when using open-ended/short answer responses on the cases. Additionally, "thinking-aloud" protocols while evaluating cases and subsequent qualitative analyses of the results may provide more refined interpretations of the clinicians' rationales and diagnostic thinking pathways. Finally, real-time testing of the scenarios would assess participants' ability to think on their feet without using aids or correcting responses following a period of reflection.

Teaching students and residents to manage medical emergencies in their offices begins with training in dental school and residency programs. A thoughtful and deliberate set of strategies to enhance best practices ought to include multiple and complementary approaches. We list four salient and innovative ideas to help implement teaching strategies to handle medical emergencies in dental 
practices. First, interacting with medical colleagues is likely to benefit the learning process for dental students and residents as many major medical campuses have simulation centers in which well-designed mannequin stations mimic medical emergencies. Dental students and residents could gain hands-on experience supported by the use of such simulation centers after appropriate adaptations. In this regard, dental schools could partner with medical centers to allow students the ability to train at these centers. Second, having dental students and residents work in groups to develop case scenarios and management plans would improve learning. According to the social constructivism tenet that human development is socially situated and knowledge is constructed through interaction with others, ${ }^{16}$ dental students and residents would not only bring their theoretical experiential knowledge to identify situations but also to create management plans. Third, schools should consider developing communities of practice ${ }^{17}$ to include medical and dental students, as well as residents, to work in teams. While embodying one aspect of interprofessional education, such a strategy could be further supported with opportunities to experience telemedicine/dentistry tools and the use of mobile applications. ${ }^{18}$ Finally, making a commitment to conducting regular emergency drills at dental schools to reinforce both simulation and group learning could enhance learning outcomes.

\section{Conclusion}

The results from this study indicated that the participants could be better prepared in the diagnosis and management of medical emergencies in dental settings. We propose that incorporating periodic emergency drills in dental settings, along with participating in CE courses, could help dentists manage medical emergencies. The focus should be on how to properly diagnose and manage cardiac arrest, anaphylaxis, syncope, and myocardial infarction in dental settings.

\section{Acknowledgments}

This study was supported by the Indiana University School of Dentistry graduate student research fund.

\section{REFERENCES}

1. Boyd BC, Fantuzzo JJ, Votta T. The role of automated external defibrillators in dental practice. N Y State Dent J 2006;72(4):20-3.
2. Muller MP, Hansel M, Stehr SN, et al. A state-wide survey of medical emergency management in dental practices: incidence of emergencies and training experience. Emerg Med J 2008;25(5):296-300.

3. Girdler NM, Smith DG. Prevalence of emergency events in British dental practice and emergency management skills of British dentists. Resuscitation 1999;41(2):159-67.

4. Al-Sebaei MO, Alkayyal MA, Alsulimani AH, et al. The preparedness of private dental offices and polyclinics for medical emergencies: a survey in Western Saudi Arabia. Saudi Med J 2015;36(3):335-40.

5. Medical emergencies. In: Brian JN, ed. Complete review of dental hygiene. Upper Saddle River, NJ: Prentice Hall, 2002:483-504.

6. Haas DA. Management of medical emergencies in the dental office: conditions in each country, the extent of treatment by the dentist. Anesth Prog 2006;53(1):20-4.

7. Johnson BR, Epstein JB. The medically complex endodontic patient. In: Ingle JL, Baumgarner JC, eds. Ingle's endondontics. Hamilton, BC: Decker, 2008:749-79.

8. Kumarswami S, Tiwari A, Parmar M, et al. Evaluation of preparedness for medical emergencies at dental offices: a survey. J Int Soc Prev Community Dent 2015;5(1):47-51.

9. Kieser J, Herbison P. Clinical anxieties among dental students. N Z Dent J 2000;96(426):138-9.

10. Fast TB, Martin MD, Ellis TM. Emergency preparedness: a survey of dental practitioners. J Am Dent Assoc 1986;112(4):499-501.

11. Anders PL, Comeau RL, Hatton M, Neiders ME. The nature and frequency of medical emergencies among patients in a dental school setting. J Dent Educ 2010;74(4):392-6.

12. Miller CS, Epstein JB, Hall EH, Sirois D. Changing oral care needs in the United States: the continuing need for oral medicine. Oral Surg Oral Med Oral Pathol Oral Radiol Endod 2001;91(1):34-44

13. Chapple ILC. Diabetes and periodontal diseases: consensus report of the joint EFP/AAP workshop on periodontitis and systemic diseases. J Periodontol 2013;84(4 Suppl):S106-12.

14. American Heart Association. 2015 American Heart Association guidelines update for cardiopulmonary resuscitation and emergency cardiovascular care. At: eccguidelines. heart.org/index.php/circulation/cpr-ecc-guidelines-2/. Accessed 29 July 2017.

15. American Dental Association. Oral health topics: medical emergencies in the dental office. At: www.ada.org/en/ member-center/oral-health-topics/medical-emergenciesin-the-dental-office. Accessed 29 July 2017.

16. Vygotsky L. Interaction between learning and development. New York: Macmillan, 2008.

17. Lave J, Wenger E. Situated learning: legitimate peripheral participation. Cambridge, UK: Cambridge University Press, 1990.

18. Ventola LC. Mobile devices and apps for health care professionals: use and benefits. Phamacol Ther 2014;39 (5):356-64 